\title{
Visualization of Learning-induced Synaptic Plasticity in Output Neurons of the Drosophila Mushroom Body $Y$-Lobe
}

\author{
Clare E. Hancock \\ University of Göttingen \\ Vahid Rostami \\ University of Cologne \\ Martin P. Nawrot \\ University of Cologne \\ André Fiala ( $\square$ afiala@gwdg.de) \\ University of Göttingen
}

\section{Research Article}

Keywords:

Posted Date: January 31st, 2022

DOI: https://doi.org/10.21203/rs.3.rs-1296152/v1

License: (c) (i) This work is licensed under a Creative Commons Attribution 4.0 International License. Read Full License 


\section{Abstract}

By learning, through experience, which stimuli coincide with dangers, it is possible to predict outcomes and act pre-emptively to ensure survival. In insects, this process is localized to the mushroom body (MB), the circuitry of which facilitates the coincident detection of sensory stimuli and punishing or rewarding cues and, downstream, the execution of appropriate learned behaviors. Here, we focused our attention on the mushroom body output neurons (MBONs) of the $y$-lobes that act as downstream synaptic partners of the MB $\mathrm{Y}$-Kenyon cells (KCs) to ask how the output of the MB $\mathrm{y}$-lobe is shaped by olfactory associative conditioning, distinguishing this from non-associative stimulus exposure effects, and without the influence of downstream modulation. This was achieved by employing a subcellularly localized calcium sensor to specifically monitor activity at MBON postsynaptic sites. Therein, we identified a robust associative modulation within only one MBON postsynaptic compartment (MBON- $\gamma 1$ pedc $>\alpha / \beta)$, which displayed a suppressed postsynaptic response to an aversively paired odor. While this MBON did not undergo non-associative modulation, the reverse was true across the remainder of the $y$-lobe, where general odor-evoked adaptation was observed, but no conditioned odor-specific modulation. In conclusion, associative synaptic plasticity underlying olfactory learning is localized to one distinct synaptic KC-to-MBON connection.

\section{Introduction}

Deciphering how and where in neuronal brain circuits learned information is acquired and stored, i.e., distributed across many neurons or localized to specific synapses, is a central topic in neuroscience. The fruit fly, Drosophila melanogaster, has proven to be a valuable model organism for testing hypotheses in this area of research. Indeed, despite their relatively modest brain size compared with mammals, fruit flies are able to perform a variety of complex learning tasks. This includes classical, Pavlovian-style conditioning ${ }^{[1]}$ in which an odor (conditioned stimulus, CS) is associated with an appetitive or aversive unconditioned stimulus (US) ${ }^{[2],[3]}$. Such associative learning forms the basis of the current study. In Drosophila, the mushroom bodies of the central brain have been identified as the site of the neural plasticity that facilitates this learning process ${ }^{[4],[5]}$. More specifically, the $y$-lobes of the mushroom bodies are required for the acquisition process and the formation of a short-term memory directly after training ${ }^{[6]-[8]}$. Consequently, not only have intrinsic mushroom body neurons (Kenyon cells, KCs) been identified as essential for associative learning, but so too have their extrinsic, axonal up- and downstream synaptic partners. Ensembles of KCs encode different odor stimuli selectively, as sparsely distributed neuronal activity ${ }^{[9]-[12]}$. Assigning behavior-instructive values to these odor representations through learning is mediated by axonal upstream US-encoding dopaminergic neurons (DANs) and downstream action-inducing mushroom body output neurons $(\mathrm{MBONs})^{[13]-[16]}$. Whereas the former signal the presence of the US at the time of conditioning ${ }^{[15],[16]}$, the latter have been identified as key players in the guiding of approach towards or repulsion away from the learned odor ${ }^{[13],[14]}$. With distinct populations of MBONs signalling positive or negative valence ${ }^{[13]}$, current models postulate that the coincident activity of the odor-coding KCs and antagonistically acting (i.e. rewarding or punishing) US-coding DANs leads to a 
shift in the relative activation of the respective antagonistically acting MBONs ${ }^{[17]}$. This implies that the balanced activity of populations of MBONs ultimately represent the behavior-instructive 'readout' of the memory trace. Coupled with findings that learning leads to a synaptically localized modulation of KC activity ${ }^{[11]}$, and that specifically the synaptic output from the KCs is required for short-term memory recall but not its acquisition [18], [19], the KC-to-MBON presynapses have emerged as the site of plasticity mediating this type of learning ${ }^{[20]-[22]}$. However, determining which exact synapses become modified through the coincidence between the CS and US (i.e., the 'memory trace' in a strict sense) has been difficult because electrophysiological or functional imaging approaches typically monitor a neuron's activity in its entirety, including all excitatory, inhibitory, and modulatory inputs. In this study, we targeted the genetically encoded calcium indicator GCaMP to the MBON postsynapse, via fusion to the postsynaptic density protein $\mathrm{dHomer}^{[23]}$ (Fig. 1). Therefore, the relative changes in fluorescence that we quantify here are reflective of changes in calcium influx directly at the postsynapse, and less so of the integrated calcium signal across the larger dendritic arborizations that lacks the precision required to address our question. We asked whether associative plasticity is distributed across different KC-to-MBON synapses along the $\mathrm{\gamma}-\mathrm{KC}$ axons or, alternatively, whether it is confined to particular axonal compartments. We found that differential aversive olfactory conditioning led to an odor-specific, associative modulation of a single MBON postsynapse innervating the $\gamma 1$ compartment. All other MBONs (innervating the $\gamma 2-5$ compartments) showed non-associative, adaptive plasticity. Therefore, we here confine the associative short-term memory trace to one synapse type along a distinct axonal compartment of the mushroom body.

\section{Methods}

\section{Fly strains}

Flies were reared on standard cornmeal food medium at $25^{\circ} \mathrm{C}$ and $60 \%$ relative humidity with a $12: 12 \mathrm{~h}$ light/dark cycle. Split-gal4 driver lines used to drive expression in MBONs were obtained from the Bloomington Drosophila Stock Centre: MB112C (BDSC \#68263, MBON-ү1pedc > a/ $\beta$ ), MB077B (BDSC

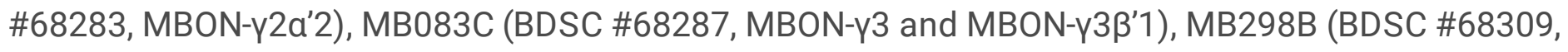
MBON- $44>\gamma 1 \gamma 2$ ), MB210B (BDSC \#68272, MBON- $\left.55 \beta^{\prime} 2 a, M B O N-\beta^{\prime} 2 m p\right)$. UAS-GCaMP6f flies were also obtained from Bloomington Drosophila Stock Centre (BDSC \#52869) ${ }^{[24]}$. UAS-dHomerGCaMP3 flies were generated by Pech et al. ${ }^{[23]}$.

\section{Immunohistochemistry}

Flies were cold anaesthetized on ice for $5 \mathrm{~min}$ before being moved to a dissection dish and fixed with insect pins. Brains were extracted in Ringer's solution $\left(5 \mathrm{mM} \mathrm{KCl}, 130 \mathrm{nM} \mathrm{NaCl}, 2 \mathrm{mM} \mathrm{MgCl} 2,2 \mathrm{mM} \mathrm{CaCl}_{2}\right.$, $5 \mathrm{mM}$ Hepes, $36 \mathrm{mM}$ sucrose, $\mathrm{pH}$ 7.3) by removing the head capsule and detaching the brain from the ventral nerve cord. Brains were then fixed in $4 \%$ paraformaldehyde at room temperature for $45 \mathrm{~min}$, and then washed three times in PBS with $0.6 \%$ Triton-X before being incubated for $2 \mathrm{~h}$ at room temperature in blocking solution containing $2 \%$ bovine serum albumin. To amplify GCaMP signal, brains were incubated 
with anti-GFP primary antibody (rabbit anti-GFP, Invitrogen A6455) at a concentration of 1:2000. Additionally, an anti-discs large (DLG) primary antibody (mouse anti-DLG, Developmental Studies Hybridoma Bank 4F3) was used at a concentration of 1:200 to visualize brain structures. Brains were incubated in primary antibody solution for 1 day at $4^{\circ} \mathrm{C}$, and washed again three times in PBS with Triton$X$ before incubation in secondary antibody. Secondary antibodies anti-chicken AlexaFluor488 and antimouse AlexaFluor633 were used, both at a concentration of 1:300, for 1 day at $4^{\circ} \mathrm{C}$. Brains were washed again three times in PBS with Triton-X, and then mounted in VectaShield for confocal scanning.

\section{In vivo calcium imaging}

Female flies aged between 3-8 days after eclosion were prepared and imaged as described by Hancock et al. ${ }^{[25]}$. Flies were cold anaesthetized on ice for no more than $5 \mathrm{~min}$, placed in a custom-built chamber, and fixed in place using clear adhesive tape. A small window was cut in the tape and the head was glued in position using blue, light-curing glue. The head cuticle was then removed in a drop of Ringer's solution using a fine-bladed knife and forceps, and excess tissue was carefully removed to expose the brain for imaging. Prepared flies were then placed downstream of a custom-built odor delivery device under a Zeiss 7MP microscope equipped with a Ti-Sapphire laser (Coherent) and a Plan-Apochromat 20X water immersion objective ( $N A=1)$. An excitation wavelength of $920 \mathrm{~nm}$ was used. Image acquisition was controlled using Zeiss Zen (2011 SP4) software. Images were captured at a framerate of $4 \mathrm{~Hz}$ and with a frame size of $512 \times 512$ pixels. Simultaneous odor delivery and image acquisition was controlled via a custom written LabView program (National Instruments). Three odors were used in all experiments, as follows: 4-methylcyclohexanol (MCH), 3-Octanol (3-Oct), and 1-Octen-3-ol (1-Oct), at concentrations of $1: 750,1: 500$, and 1:400, respectively, in mineral oil.

To induce and monitor the effects of aversive olfactory conditioning, a three-stage imaging protocol was used. First, flies were sequentially presented with the three odorants and changes in fluorescence were monitored. Second, the flies were exposed to one of three conditioning protocols. Third, the flies were again presented with the three odorants and their responses were measured (see also the schematic illustration in Fig. 3). The three conditioning protocols were as follows: classical aversive associative conditioning ('paired'), in which one odor (the CS+, either $\mathrm{MCH}$ or 3-Oct) was presented for $60 \mathrm{sec}$ while a pulsing electric shock was delivered to the legs and thorax of the fly (twelve $90 \mathrm{~V}$ shocks over $60 \mathrm{sec}$, each lasting $1.25 \mathrm{sec}$ ), followed by a $60 \mathrm{sec}$ break, and then a $60 \mathrm{sec}$ presentation of a second odor (the $\mathrm{CS}$-, either $\mathrm{MCH}$ or 3-Oct) without the electric shock; an 'odor only' control, in which the same procedure was presented with the omission of the electric shock; or a 'shock only' control, in which only the electric shock stimulus was delivered, with no odors. In the first and third stages (the 'pre-training' and 'posttraining' imaging steps), all odor presentations had a duration of $2.5 \mathrm{sec}$ and were separated by an interval of approximately $40 \mathrm{sec}$.

Images were processed using ImageJ/Fiji (National Institutes of Health, $\mathrm{NIH}$ ). Any small movements were corrected using the TurboReg plugin ${ }^{[26]}$. Regions of interest (ROIs) were drawn manually to encompass the entire dendritic compartment for each MBON. The fluorescent intensity throughout the recording 
period was then extracted from these ROls and used to calculate the normalized relative change in fluorescence over time $\left(\Delta F / F_{0}\right)$ for each recording. For all measurements, $F_{0}$ was calculated as the average fluorescence intensity over the $2 \mathrm{sec}$ preceding odor delivery, and $\Delta \mathrm{F}$ was calculated by subtracting this value at each time point.

\section{Population decoding}

We devised a supervised machine learning approach to predict the CS+ vs. CS- condition from the neural ensemble activity across all five MBONs in the associative conditioning protocol. To this end, we used the area under the curve (AUC) for each $\Delta \mathrm{F} / \mathrm{F}_{0}$ time series, normalized to the mean of all data of the same odorant, which left us with 10 data samples per neuron type during post-training. For the pre-training data, we pooled the data across the three experimental protocols to increase the sample size. We then performed a binary classification task between two conditions defined by either CS+ or CS- presentation, constructing the training and test data as follows: For each condition, we randomly selected one data sample from each neuron type to construct the test set, which represented the responses of a pseudo population ${ }^{[27]}$ with all five MBON types for each $y$-lobe compartment. From the remaining samples, we created the training set by randomly sampling, for each condition, complete sets of five MBONs with replacement ( $N_{\text {train }}$ random combinations per condition). We repeated the process of randomly assigning

a test set $N_{\text {test }}$ times to ensure that the analysis was not biased based on the choice of neurons/animals in the test set. Then, we trained a support vector machine classifier (SVM) to distinguish between two experimental conditions. We then evaluated the performance of each classifier by calculating the accuracy score across test sets in percent. Classification during pre-training served as a control with an expected accuracy at the level of chance (50\%). All analysis was carried out with Python and the implementation of SVM in the Scikit-learn package ${ }^{[28]}$. As further controls, we applied the same classification to the post-training data samples in the experimental protocols of 'odor only' and 'shock only'. We created two classes, $A$ and $B$, through the random assignment of odors to those labels.

\section{Statistical analyses}

Odor-induced calcium dynamics throughout are presented as line graphs, with lines representing mean $\Delta \mathrm{F} / \mathrm{F}_{0}$ values over time, and shaded areas representing the standard error of the mean (SEM). To quantify and compare responses, the integrated $A U C$ for each $\triangle F / F_{0}$ time series was calculated over the $5 \mathrm{sec}$ after odor onset. Tests for significant changes in pre-to-post training AUC were carried out using Wilcoxon signed rank tests. For testing whether the accuracy with which the classifier detected trained from not trained odor responses, one sample Wilcoxon signed rank tests against the level of chance (50\%) were used.

\section{Results}

\section{Visualization of postsynaptic calcium in mushroom body output neurons}


Acquisition and formation of an associative short-term memory is localized to the KC presynapses of the

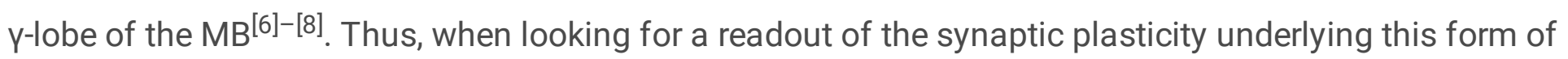
memory, we focused on the population of compartment-specific MBONs that lie directly downstream of the $\mathrm{Y}$-type KCs. These neurons each receive synaptic input from KCs in their respective compartment, the weight of which is modulated by input from DANs and comprises the canonical site of learning-induced plasticity within this circuit (Fig. 1a-c) ${ }^{[14],[20]-[22]}$. In addition, MBONs receive direct modulatory input from DANs as well (Fig. 1c). Therefore, we utilized a postsynaptically localized tool, $d$ Homer-fused GCaMP3 ${ }^{[23]}$, that allowed for the monitoring of odor-evoked activity precisely at the site of MBON input. To first verify the localization of $d$ Homer-GCaMP ${ }^{[23]}$ to the postsynaptic compartments of the MBONs, we removed the brains of flies expressing this construct in individual MBONs of the mushroom body $y$-lobe and subjected them to immunohistochemistry and confocal microscopy. When compared to the cytosolic GCaMP6f, dHomer-GCaMP results in a spatially restricted, overall more punctuated fluorescence that is localized primarily in the dendritic compartments of neurons (Fig. 1d). This difference was also observed when neurons were visualized in vivo using two-photon microscopy (Fig. 1e and f), with the large neurites that predominate the GCaMP6f fluorescence being absent in dHomer-GCaMP-expressing flies (the $\gamma 1$ compartment innervation by MBON- $\gamma 1$ pedc $>\alpha / \beta$ is shown here as an example). To further verify that the latter can also be used to detect postsynaptic odor-evoked activity in MBONs, flies were presented with $\mathrm{MCH}$ and 3-Oct and changes in fluorescence were quantified (Fig. 1e and f). Indeed, reflective of the less intense overall fluorescence, postsynaptic odor responses monitored using $d$ Homer-GCaMP were lower in magnitude than the gross calcium signal observed using GCaMP6f, although still robust enough to be reliably detected and analyzed. With this, we validate $d$ Homer-GCaMP as a viable tool for studying localized odor representations at the level of the MBON postsynapse.

\section{Y-lobe MBONs receive heterogeneous odor-evoked inputs}

Congruent with their receipt of inputs from large populations of KCs, previous studies have shown that MBONs innervating the MB lobes have broad odor response profiles ${ }^{[29]}$. In this vein, we first sought to analyze the postsynaptic responsiveness of our $\mathrm{Y}-\mathrm{MBONs}$ of interest to the experimental odors, $\mathrm{MCH}$ and 3-Oct. To do so, we carried out in vivo functional imaging of individual female flies expressing $d$ HomerGCaMP in each of the MBONs and quantified the odor-evoked changes in postsynaptic calcium (Fig. 2; individual and mean response traces shown in Supplementary Fig. 1).

This analysis revealed that, indeed, in the case of the MBONs innervating the $\gamma 1, \gamma 3$, and $\gamma^{4}$ compartments, robust responses to both odors were observable in the majority of flies measured (Fig. 2a, c, d). Surprisingly, the $\gamma 2$-innervating MBON ( $\left.\mathrm{Y}^{2} \mathrm{a}^{\prime} 1\right)$ only responded to 3-Oct in a small number of flies, in contrast to $\mathrm{MCH}$ (Fig. 2b). It has previously been reported that the dendritic arbors of MBONs in the $y 5$ compartment (MBON- $\gamma 5 \beta^{\prime} 2 \mathrm{a}, \mathrm{MBON}-\beta^{\prime} 2 \mathrm{mp}$ ) show little or no response to olfactory stimuli ${ }^{[30]}$. We also observed this result, with responses being detected in only $~ 17 \%$ of flies measured (Fig. 2e, Supplementary Fig. 1). Differences in the magnitude of responses are also notable, with those measured at the MBON- $\gamma 3 / \mathrm{MBON}-\gamma 3 \beta^{\prime} 1$ postsynapse sometimes being 4-5x greater than those measured in, for example, MBON- $\gamma 1$ pedc $>\alpha / \beta$. These differences in magnitude were also be observed between odors 
within animals, with $\mathrm{MCH}$ eliciting stronger responses than 3-Oct in most cases. These findings indicate that, first, olfactory responses between the $\mathrm{Y}$-lobe MBONs and, thus, the efficiency of KC-to-MBON connections in different $y$-lobe compartments, are not homogenous. Second, within MBON types, responses to different odors are not homogenous across individuals. Therefore, we confirm that the neurons in this MBON population possess distinct and individualized odor response properties that could indeed influence and/or result from individual experience such as olfactory learning, as already reported using a cytosolic calcium sensor ${ }^{[29]}$.

\section{Associative conditioning gives rise to compartment- specific plasticity}

To investigate if and how these odor representations are influenced by associative learning, we subjected the same flies expressing $d$ Homer-GCaMP in the $y$-lobe MBONs to an aversive conditioning protocol under the microscope and monitored odor-evoked postsynaptic calcium. Flies were placed in a custombuilt imaging chamber in which they could be exposed to both odor and electric shock stimuli during functional imaging, allowing for the visualization of odor-evoked postsynaptic activity before and after an aversive training in which flies learned to associate a given odor with punishment ${ }^{[25]}$.

Based on the hypothesis that the combinatorial activity of the $y$-lobe MBONs holds behavior-instructive information about learned odor valence, we first hypothesized that aversive associative conditioning would lead to bidirectional modulation of MBONs such that approach-mediating MBONs would be suppressed in response to the aversively paired odor and avoidance-mediating MBONs would be potentiated. Indeed, such effects have been reported when measuring with cytosolic calcium indicators or using electrophysiology ${ }^{[30]-[32]}$. In the following sections, however, we demonstrate that modulation directly at the MBON postsynapse is a highly specialized occurrence, localized to a singular compartment of the $y$-lobe.

Pairing of an odor with electric shock led to a significant reduction in the postsynaptic calcium response elicited by the trained odor (CS+) in MBON- $\gamma 1$ pedc $>\alpha / \beta$ (Wilcoxon signed rank test, $Z=2.01399$, $p=0.03906$ ) (Fig. 3c, left). This is consistent with the identification of this neuron in the signaling of positive stimulus valence [12]. Neither the CS- odor (that was explicitly not paired with electric shock) nor the control odor (1-Oct) elicited statistically significant changes in response after conditioning (CS-: Wilcoxon signed rank test, $Z=0, p=1$; control odor: Wilcoxon signed rank test, $Z=0.82929, p=0.42578$ ) (Fig. 3c, center and right). This was also the case in the control groups for odor presentation, but without any electric shock ('CS only' control; Fig. 3d). This finding demonstrates association-specific modulation of the odor-driven inputs to MBON- $\gamma 1$ pedc $>\alpha / \beta$, dependent on CS-US contiguity. Interestingly, the stimulation with electric shock, but without any odor presentation ('US only control'; Fig. 3e) induced a slight, but not statistically significant increase in MBON- $\gamma 1$ pedc $>\alpha / \beta$ response at the given sample size. However, when data for both odorants that were also used for associative training, $\mathrm{MCH}$ and 3-Oct, were pooled, a statistically significant increase was detected (Supplementary Fig. 3) (Wilcoxon signed rank test, $Z=-2.55729 ; p=0.00831)$. This illustrates that the postsynaptic calcium response in this particular 
MBON can be bidirectionally modulated; it decreases in response to an odor associated with punishment, and it increases in response to an odor if the punishment does not occur in temporal coincidence with it.

Conversely, no association-induced changes were observable in MBONs of the remaining $y$-lobe compartments (Fig. 4). Rather, a strong decrease in odor-evoked calcium activity occurred between the pre- and post-training odor response measurements in MBONs innervating the $\gamma 3$ and $\gamma 4$ compartments, but both to the CS+ and CS- odor (Fig. 4a, c, e, g). The y5-innervating MBONs represent an exception in that, in most cases, they showed no responses to odors throughout experiments (Fig. $4 \mathrm{~g}$ ). In MBONs innervating the $\mathrm{\gamma} 2$ compartment, this effect was not statistically significant, perhaps because of relatively weak odor-evoked calcium activity before training in this group of animals (Fig. 4a). The relatively high variability in odor-evoked calcium activity across individuals is in accordance with previous reports that suggest highly individualized, perhaps experience-dependent responsiveness in MBONs innervating the lobes ${ }^{[29]}$. Flies that received the 'odor only' control procedure also displayed strong reductions in responses (Fig. 4b, d, f). Strikingly, the previously high amplitude responses in the $\gamma^{3}$-innverating MBON are almost entirely lost through either of these protocols (Fig. 4c, d). In most cases, this adaptation is not odor-identity specific and is generalized to the third odor, 1-Oct, that is not presented during training (Supplementary Fig. 2). We conclude that this adaptation is likely caused by the prolonged odor exposure that occurs during both the conditioned (paired) and the 'odor only' control protocols, as adaptation is much weaker in flies that received the 'shock only' control procedure (Supplementary Fig. 3).

These results go beyond confirmation that the MBONs of the $y$-lobe show differential naïve odor responses ${ }^{[29]}$, and that the manner in which those odor responses are modulated by dopamine is diverse across different $\mathrm{MBONs}^{[33]}$. Our data indicate that experience-dependent changes in odor-evoked, postsynaptic calcium activity occur in MBONs $\mathrm{Y}_{1}-\mathrm{\gamma} 4 \mathrm{MBONs}$. However, a differential modulation resulting from CS-US coincidence is restricted to the $\gamma 1$ compartment. Therefore, the synaptic DAN-KCMBON microcircuitry that mediates the CS-US association process during aversive olfactory conditioning (i.e., the memory trace in a strict sense) is confined to a single $y$-lobe compartment and not distributed across different compartments.

\section{MBON responses are indicative of whether an odor has been aversively trained or not.}

Given this finding, we sought to test in an unbiased manner whether these observed changes in postsynaptic calcium responses are actually indicative of whether the odor-evoked calcium transients have been aversively trained or not. To do so, we applied a machine learning approach, and used pseudopopulations of $\gamma 1-\gamma 5 \mathrm{MBON}$ responses to train a classifier. Over multiple training sessions, the classifier was provided with the pre- or post-training odor responses of such pseudo populations of $y$-lobe MBONs and was then asked to predict whether the response was from one of two conditions, CS+ or CS-. On average, the conditioned odor (CS+) was distinguished with significantly greater accuracy than any other condition (one sample Wilcoxon sign rank test against level of chance (50\%), Z=16.78943, $p<0.0001$ ). (Fig. 5a) Indeed, all other experimental conditions (pre-training, 'odor only', or 'shock only' conditions), resulted in an approximate $50 \%$ success rate (one sample Wilcoxon sign rank test against level of chance 
(50\%), pre-training: $Z=1.7353, p=0.08269$, 'odor only' control: $Z=0.19191, p=0.84782$, 'shock only' control: $Z=1.15577, p=0.24778$ ), indicating that the odor representations that we observed at the MBON postsynapse are in fact indicators of whether the odor has been trained as aversive or not. We then compared the accuracy with which the classifier could distinguish between CS- and CS+ odors after training in a situation in which one of the five $y$-lobe MBON types was removed from the training data sets. Only removing the MBON innervating the $\gamma 1$ compartment decreased the accuracy of differentiating $\mathrm{CS}+$ from CS- to the level of chance (Fig. 5b) (one sample Wilcoxon signed rank test against level of chance $(50 \%), Z=1.77029, p=0.07668)$. Excluding any other MBON type did not significantly affect the accuracy of discriminability (Fig. 5b) (one sample Wilcoxon signed rank test against level of chance (50\%), $\gamma 2$ excluded: $Z=16.39812, p<0.0001, \gamma 3$ excluded: $Z=9.87016, p<0.0001, \gamma 4$ excluded: $Z=10.72866$, $p<0.0001$, $\gamma 5$ excluded: $Z=13.54734, p<0.0001)$, corroborating the finding of the $\gamma 1$ compartment as the site of differential synaptic plasticity underlying aversive discrimination learning.

\section{Discussion}

Determining the substrate mediating the acquisition and storage of learned information through the properties of single neurons, their synapses, and the neural circuits the neurons are part of is a key challenge in neuroscience. Here, we do not define the term 'memory trace' as all potential physiological changes within neurons, synapses, and the entire neuronal circuits that accompany or result from the learning process. Considering that plastic changes in synaptic transmission are the principal substrate underlying learning and memory formation ${ }^{[34]-[37]}$, we define a memory trace as those synaptic changes that are required and sufficient to induce the learned response ${ }^{[35],[37]}$. In the case of classical conditioning, these synaptic changes must be the site of CS-US integration. Decades of research have established that a) axonal KC presynapses are required for associative odor learning and short-term

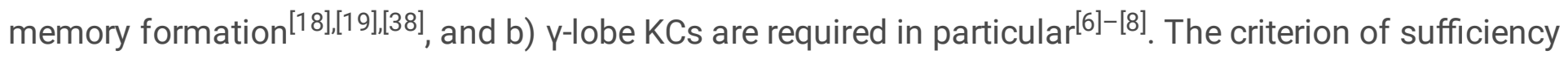
is difficult to address because the stochastic and sparsely distributed nature of odor representations across all KCs precludes any artificial activation of a selected $\mathrm{KC}$ and subsequent test of whether an odor stimulus has been learned. However, artificial activation of defined KCs in coincidence with electric shocks has been used to show that a particular KC activity pattern can be trained, and its subsequent

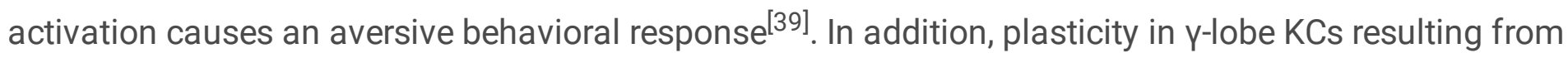
associative learning has been clearly demonstrated ${ }^{[11],[40]}$.

However, genetic manipulation of neurons (e.g., using transgenes to depolarize or hyperpolarize neuronal membranes or to block synaptic transmission) inevitably affects entire cells; the question of which dendritic or axonal part of a neuron is required or sufficient for mediating learning remains unanswered using such approaches. This is of importance because the functional compartmentalization of $\mathrm{KC}$ axons, with each compartment being innervated by distinct subsets of DANs mediating reward or punishment, and MBONs inducing different behavioral actions ${ }^{[13],[14]}$, suggests that entire neurons are not the functional units representing the substrate of memory traces (such as 'engram cells'). Rather, subcellular 
partitions of neurons, in this case partitions of axons spanning a compartment, represent independently modulated functional units ${ }^{[11]}$.

Previous functional imaging and electrophysiological studies recording from neurons in their entirety (i.e., measurements at the soma level), have also reported bidirectional modulation of MBONs as a result of associative olfactory conditioning ${ }^{[29]-[31]}$. We conclude from our data that plasticity in these neurons arises downstream of the KC-to-MBON synapse via previously identified feedforward and feedback loops that exist in the MB circuit. For example, it was shown that plasticity in the specific KC > MBON-y1 pedc > $\alpha / \beta$ connection does not remain confined to the $\gamma 1$ compartment, but causes subsequent downstream

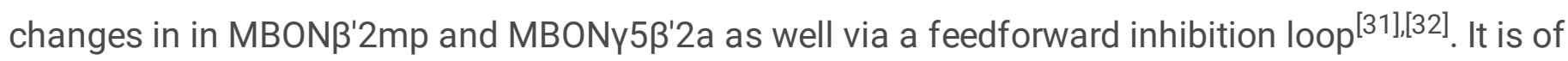
course important to note here that although the vast majority of inputs to MBONs arise from KCs, MBONs receive inputs at various dendritic regions from diverse cell types (including DANs and other MBONs). Our approach to confine the measurement to MBON postsynapses might therefore contribute to distinguishing the primary effects of the CS-US association (i.e., the 'memory trace') and subsequent downstream effects that result from it ${ }^{[41]}$. Importantly, we used odor stimuli and electric shocks as unconditioned stimuli, as they are typically applied in behavioral learning experiments; this approach is in contrast to optogenetic stimulation of selected DANs ${ }^{[16],[33]}$ that may or may not reflect real sensory stimulation.

Our results directly support recent computational model studies. A circuit model by Springer and Nawrot ${ }^{[42]}$ assumed downregulation of the synaptic input from KCs to MBON- $\gamma 1$ pedc $>\alpha / \beta$ through a plasticity mechanism that requires pairing of the CS+ odor stimulus with neuromodulatory input from the aversive US-signaling PPL1- $y 1$ pedc DAN. This simulation showed a significant reduction in MBON$\gamma 1$ pedc $>\alpha / \beta$ synaptic input and avoidance behavior after a single pairing, as confirmed in our experiments (Fig. 3). In this model, feedforward inhibition from MBON- $\gamma 1$ pedc $>\alpha / \beta$ to MBON- $\gamma 5 \beta^{\prime} 2 a$ and feedback excitation to dopaminergic neurons establishes a prediction-error mechanism that supports the saturation of the behavioral learning curve and enables extinction learning. Similarly, a recent model by Bennet et al. ${ }^{[43]}$ assumed the explicit downregulation of the synaptic weight between $\mathrm{KCs}$ and an unspecified approach-mediating MBON to implement aversive conditioning and reward prediction through positive MBON-DAN feedback. In fact, recent advances in connectomics have revealed the presence of numerous, multilayered feedback motifs in and around the MB that are implicated in learning and memory formation (see ${ }^{[44]-[46]}$ ). Of course, our data do not rule out the possibility for associative learning-induced modulation of other MBONs in other forms of learning, e.g., over longer time periods, conditioning of different valences such as reward, or context. For example, it is well-established that longterm memory formation requires $\mathrm{KCs}$ of the vertical $\mathrm{a} / \mathrm{a}^{\prime}-\mathrm{Iobes}^{[47]}$. It must be pointed out that our data refer only to the acquisition process and immediate short-term memory formation of aversive olfactory learning. The formation of longer-lasting memories might be initiated in parallel and independently.

The broad and generalized suppression of odor responses in all more distal MBON postsynapses indicates that they, unlike MBON- $\gamma 1$ pedc $>\alpha / \beta$, possess properties more tuned to signaling non- 
associative events, such as adaptation resulting from prolonged odor stimulation. Two key observations indicate that the non-associative changes manifested principally as strong reductions in odor-evoked postsynaptic calcium responses in post-training recordings in the majority of MBONs (namely: MBON-

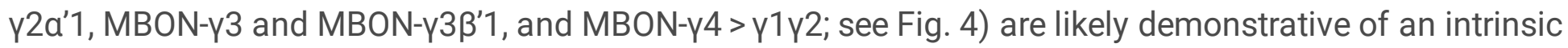
adaptive property of these MBONs, rather than merely sensory adaptation. First, the observed adapations are not odor specific, but instead are generalized across all odors (Fig. 4, Suppleentary Fig. 2). Second, adaptation in the upstream olfactory pathway would theoretically lead to adapation across all MBONs, which we do not observe (MBON- $\gamma 1$ pedc $>\alpha / \beta$ displayed no adaptation in any context; Fig. 3 ). This further highlights MBON- $\gamma 1$ pedc $>\alpha / \beta$ as functionally distinct from the remaining $\gamma$-lobe MBONs investigated here. The non-associative effects observed here are yet to be included in future computational circuit models of the fly MB. At the population level of KCs, synaptic plasticity induced by associative olfactory learning causes decorrelation of odor-evoked calcium influx at axonal synaptic boutons, and across the axonal compartments $\gamma 2-\gamma 5^{[11]}$; axonal $\mathrm{KC}$ boutons in the $\gamma 1$ compartment could not be recorded due to technical limitations, which leaves open the question of whether axonal boutons in this compartment similarly change their correlated activity. However, the fact that differential training affects odor coding across multiple compartments across KC populations differentially implies that the learned change in odor-coding of mushroom body circuit and the learned behavior-instructive properties of the MBON signals are two distinct aspects of associative learning that can be experimentally dissociated. We would like to point out that our finding that associative learning-induced changes in synaptic transmission is precisely confined to one axonal compartment does not exclude roles of other

compartments in guiding learned behavior ${ }^{[11,48]}$. However, our data suggest that the associative learning process is initiated through precisely confined plasticity at the axonal $Y 1$ compartment and the respective KC-to-MBON synapse, and that other MB lobe compartments and MBONs are subsequently affected through downstream processes.

\section{Declarations}

\section{Acknowledgements}

We are grateful to the Janelia Research Campus and the Bloomington Drosophila stock center for providing Drosophila strains. We thank El Yazid Rachad for help with data analysis and the generation of figures. This work was supported by the German Research Foundation through the Research Unit 'Structure, Plasticity and Behavioral Function of the Drosophila Mushroom Body' (FOR 2705) to A.F. and M.N., and through SFB 889/B4 to A.F.

\section{Data availability statement}

All original raw data are available at http://hdl.handle.net/21.11124/For2705_000092.

\section{Additional information}

The authors declare no competing interests. 
$\mathrm{CH}$ performed experiments, $\mathrm{VR}$ and $\mathrm{MN}$ performed computational analysis, $\mathrm{AF}$ and $\mathrm{CH}$ designed experiments, analyzed data, and wrote the manuscript with the help of all authors.

\section{References}

1. Pavlov, I. P. Conditioned reflexes: An investigation of the physiological activity of the cerebral cortex. Oxford Uni. Press (1927).

2. Tempel, B. L., Bonini, N., Dawson, D. R., \& Quinn, W. G. Reward learning in normal and mutant Drosophila. Proc. Natl. Acad. Sci. <bvertical-align:super;>80</bvertical-align:super;>(5), 1482-1486 (1983).

3. Tully, T., \& Quinn, W. G. Classical conditioning and retention in normal and mutant Drosophila melanogaster. J. Comp. Physiol. 157(2), 263-277 (1985).

4. de Belle, J. S., \& Heisenberg, M. Associative odor learning in Drosophila abolished by chemical ablation of mushroom bodies. Science 263(5147), 692-695 (1994).

5. Heisenberg, M., Borst, A., Wagner, S., \& Byers, D. Drosophila mushroom body mutants are deficient in olfactory learning. J. Neurogenet. 2(1), 1-30 (1985).

6. Zars, T., Fischer, M., Schulz, R., \& Heisenberg, M. Localization of a short-term memory in Drosophila. Science $\mathbf{2 8 8}$,<bvertical-align:super;> </bvertical-align:super;>672-675 (2000).

7. Blum, A. L., Li, W., Cressy, M., \& Dubnau, J. Short-and long-term memory in Drosophila require cAMP signaling in distinct neuron types. Curr. Biol. 19(16), 1341-1350 (2009).

8. Qin, H., Cressy, M., Li, W., Coravos, J. S., Izzi, S. A., \& Dubnau, J. Gamma neurons mediate dopaminergic input during aversive olfactory memory formation in drosophila. Curr. Biol. 22(7), 608614 (2012).

9. Perez-Orive, J., Mazor, O., Turner, G. C., Cassenaer, S., Wilson, R. I., \& Laurent, G. Oscillations and sparsening of odor representations in the mushroom body. Science 297, 359-365 (2002).

10. Honegger, K. S., Campbell, R. A. A., \& Turner, G. C. Cellular-resolution population imaging reveals robust sparse coding in the Drosophila mushroom body. J. Neurosci. 31(33), 11772-11785 (2011).

11. Bilz, F., Geurten, B. R. H., Hancock, C. E., Widmann, A., \& Fiala, A. Visualization of a distributed synaptic memory code in the Drosophila brain. Neuron 106(6), 963-976 (2020).

12. Betkiewicz, R., Lindner, B., \& Nawrot, M.P. Circuit and cellular mechanisms facilitate the transformation from dense to sparse coding in the insect olfactory system. eNeuro 7(2), ENEURO.0305-18.2020 (2020).

13. Aso, Y. et al. Mushroom body output neurons encode valence and guide memory-based action selection in Drosophila. eLife 3(3), e04580 (2014a).

14. Aso, Y. et al. The neuronal architecture of the mushroom body provides a logic for associative learning. eLife 3, e04577 (2014b). 
15. Riemensperger, T., Völler, T., Stock, P., Buchner, E., \& Fiala, A. Punishment prediction by dopaminergic neurons in Drosophila. Curr. Biol. 15(21), 1953-1960 (2005).

16. Cohn, R., Morantte, I., \& Ruta, V. Coordinated and compartmentalized neuromodulation shapes sensory processing in Drosophila. Cell 163(7), 1742-1755 (2015).

17. Owald, D., Felsenberg, J., Talbot, C. B., Das, G., Perisse, E., Huetteroth, W., \& Waddell, S. Activity of defined mushroom body output neurons underlies learned olfactory behavior in Drosophila. Neuron 86(2), 417-427 (2015).

18. Dubnau, J., Grady, L., Kitamoto, T., \& Tully, T. Disruption of neurotransmission in Drosophila mushroom body blocks retrieval but not acquisition of memory. Nature 411(6836), 476-480 (2001).

19. McGuire, S. E., Le, P. T., \& Davis, R. L. The role of Drosophila mushroom body signaling in olfactory memory. Science 293(5533), 1330-1333 (2001).

20. Heisenberg, M. Mushroom body memoir: From maps to models. Nat. Rev. Neurosci. 4(4), 266-275 (2003).

21. Zhang, S., \& Roman, G. Presynaptic inhibition of gamma lobe neurons Is required for olfactory learning in Drosophila. Curr. Biol. 23(24), 2519-2527 (2013).

22. Hige, T., Aso, Y., Modi, M. N., Rubin, G. M., \& Turner, G. C. Heterosynaptic plasticity underlies aversive olfactory learning in Drosophila. Neuron 88(5), 985-998 (2015a).

23. Pech, U., Revelo, N. H., Seitz, K. J., Rizzoli, S. O., \& Fiala, A. Optical dissection of experience-dependent pre- and postsynaptic plasticity in the Drosophila brain. Cell Rep. 10(12), 2083-2095 (2015).

24. Chen, T.-W., Wardill, T. J., Sun, Y., Pulver, S. R., Renninger, S. L., Baohan, A., Schreiter, E. R., Kerr, R. A., Orger, M. B., Jayaraman, V., Looger, L. L., Svoboda, K., \& Kim, D. S. Ultrasensitive fluorescent proteins for imaging neuronal activity. Nature 499, 295 (2013).

25. Hancock, C. E., Bilz, F., \& Fiala, A. In vivo optical calcium imaging of learning-induced synaptic plasticity in Drosophila melanogaster. J. Vis. Exp. 152, e60288. (2019).

26. Thévenaz, P., Ruttimann, U. E., \& Unser, M. A pyramid approach to subpixel registration based on intensity. IEEE Trans. Image Process. 7(1), 27-41 (1998).

27. Rickert, J., Riehle, A., Aertsen, A., Rotter, S., \& Nawrot, M. P. Dynamic encoding of movement direction in motor cortical neurons. J. Neurosci. 29(44), 13870-13882 (2009).

28. Pedregosa, F. et al. Scikit-learn: Machine learning in Python. J. Mach. Learn. Res. 12(85), 2825-2830 (2011).

29. Hige, T., Aso, Y., Rubin, G. M., \& Turner, G. C. Plasticity-driven individualization of olfactory coding in mushroom body output neurons. Nature 526, 258-262 (2015b).

30. Berry, J. A., Phan, A., \& Davis, R. L. Dopamine neurons mediate learning and forgetting through bidirectional modulation of a memory trace. Cell Rep. 25(3), 651-662 (2018).

31. Felsenberg, J. et al. Integration of parallel opposing memories underlies memory extinction. Cell 175(3), 709-722 (2018). 
32. Perisse, E., Owald, D., Barnstedt, O., Talbot, C. B. B., Huetteroth, W., \& Waddell, S. Aversive learning and appetitive motivation toggle feed-forward inhibition in the Drosophila mushroom body. Neuron 90(5), 1086-1099 (2016).

33. Aso, Y., \& Rubin, G. M. Dopaminergic neurons write and update memories with cell-type-specific rules. eLife<bvertical-align:super;></bvertical-align:super;>5, e16135-e16135 (2016).

34. Magee, J. C., \& Grienberger, C. Synaptic plasticity forms and functions. Annu. Rev. Neurosci. 43(1), 95-117 (2020).

35. Martin, S. J., Grimwood, P. D., \& Morris, R. G. Synaptic plasticity and memory: An evaluation of the hypothesis. Annu. Rev. Neurosci. 23, 649-711 (2000).

36. Takeuchi, T., Duszkiewicz, A. J., \& Morris, R. G. M. The synaptic plasticity and memory hypothesis: Encoding, storage and persistence. Philos. Trans. R. Soc. B. 369(1633) (2014).

37. Gerber, B., Tanimoto, H., \& Heisenberg, M. An engram found? Evaluating the evidence from fruit flies. Curr. Opin. Neurobiol. 14(6), 737-744 (2004).

38. Schwaerzel, M., Heisenberg, M., \& Zars, T. Extinction antagonizes olfactory emory at the subcellular level. Neuron 35(5), 951-960 (2002).

39. Vasmer, D., Pooryasin, A., Riemensperger, T., \& Fiala, A. Induction of aversive learning through thermogenetic activation of Kenyon cell ensembles in Drosophila. Front. Behav. Neurosci. 8(174) (2014).

40. Boto, T., Louis, T., Jindachomthong, K., Jalink, K., \& Tomchik, S. M. Dopaminergic modulation of CAMP drives nonlinear plasticity across the Drosophila mushroom body lobes. Curr. Biol. 24(8), 822831 (2014).

41. Fiala, A., and Riemensperger, T. Localization of a memory trace: aversive associative olfactory learning and short-term memory in Drosophila in Learning and Memory: A Comprehensive Reference, 2nd Edn. (ed. J. H. Byrne and R. Menzel) 475-482 (Elsevier: Academic Press, 2017).

42. Springer, M., \& Nawrot, M. P. A mechanistic model for reward prediction and extinction learning in the fruit fly. ENeuro <bvertical-align:super;>8</bvertical-align:super;>(3), ENEUR0.0549-20.2021 (2021).

43. Bennett, J. E. M., Philippides, A., \& Nowotny, T. Learning with reinforcement prediction errors in a model of the Drosophila mushroom body. Nat. Comm. 12(1), 2569 (2021).

44. Eichler, K. et al. The complete connectome of a learning and memory centre in an insect brain. Nature 548(7666), 175-182 (2017).

45. Li, F. et al. The connectome of the adult Drosophila mushroom body provides insights into function. elife 9, e62576 (2020).

46. Takemura, S. et al. A connectome of a learning and memory center in the adult Drosophila brain. eLife 6, 1-43 (2017).

47. Pascual, A., \& Préat, T. Localization of long-term memory within the Drosophila mushroom body. Science 294(5544), 1115-1117 (2001). 
48. Ichinose, T., Kanno, M., Wu, H., Yamagata, N., Sun, H., Abe, A. \& Tanimoto, H. Mushroom body output differentiates memory processes and distinct memory-guided behaviors. Curr. Biol. 31, 1294-1302 (2021).

\section{Figures}

\section{Figure 1}

Visualization of postsynaptic calcium in the mushroom body output neurons. (a) Mushroom body structure and compartmentalization of the $y$-lobe. (b) Functional compartmentalization of the $y$-lobe. (c) Putative connectivity within the Kenyon cell (KC)-dopaminergic neuron (DAN)-mushroom body output neuron (MBON) microcircuit in the $y$-lobe. (d) Representative maximum projections showing the expression pattern of cytosolic GCaMP6f and postsynaptically localized homer-GCaMP across the MBONs of the $y$-lobe. (e) and (f) Examples of functional imaging recordings using cytosolic GCaMP6f and postsynaptically localized homer-GCaMP. Line graphs show mean $\triangle F / F_{0}$ values and SEM. Grey bars indicate the odor presentation period. For GCaMP6f, $n=119$ flies, for homer-GCaMP, $n=30$ flies.

\section{Figure 2}

Odor-evoked responses at the MBON postsynapse. Heatmaps show false-color coded $\triangle F / F_{0}$ values over time, measured in each of the $\gamma$-lobe MBONs (a: MBON- $\gamma 1$ pedc $>\alpha / \beta ;$; : MBON- $\gamma 2 a^{\prime} 1 ;$; : MBON- $\gamma 3$ and

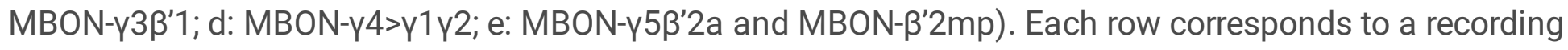
of an individual neuron in an individual fly in response to a single odor presentation. Values above heatmaps show the percentage of flies that showed a response to that odor in each MBON. Grey bars indicate the odor delivery period. For $a, c, d$, and e, $n=29$; for $b, n=30$. Schematic diagrams and single frame in vivo functional imaging examples on the left show the dendritic region of each MBON that was imaged. The green dotted line shows the region that was quantified.

\section{Figure 3}

Induction and visualization of learning-induced plasticity at the MBON- $\gamma 1$ pedc $>\alpha / \beta$ postsynapse. (a) Imaging procedures used to probe the effects of associative conditioning on MBON odor responses. Flies 
were exposed to one of three protocols: i) aversive associative conditioning; ii) 'odor only' control; or iii) 'shock only' control. Before and after the training procedure, three odors were presented and MBON postsynaptic calcium was monitored using homer-GCaMP. (b) Schematic highlighting the $\gamma 1$ compartment in which MBON- $\gamma 1$ pedc $>\alpha / \beta$ postsynaptic sites were imaged. (c-e) MBON- $\gamma 1$ pedc $>\alpha / \beta$ odor responses measured before (pre-training) and after (post-training) one of the three training protocols. Lines indicate means, and shaded areas represent SEMs. The black line in each case represents the pretraining response. Post-training responses are shown in color, dependent on condition. The yellow box indicates the period of odor presentation. The area under the curve (AUC) was calculated over the $5 \mathrm{sec}$ after odor onset, and pre- to post-training differences were tested using Wilcoxon signed rank tests ( $\left.{ }^{*} \mathrm{p}<0.05\right)$. Boxes represent $25 \%$ and $75 \%$ quartiles, squares indicate means, and horizontal lines indicate medians. Whiskers show minimum and maximum values. For the paired group (c), n=9 flies. For both control groups ( $d$ and $e), n=10$ flies.

\section{Figure 4}

Non-associative plasticity at MBON postsynapses in $\mathrm{Y2,3}$, and 4 compartments. Odor responses before and after either aversive associative conditioning $(a, c, e, g)$ or a control protocol $(b, d, f, h)$ in which the unconditioned stimulus was omitted (training protocols, top). In each case, pre-training responses are depicted in black (line graphs) or gray (box plots), and post-training responses are shown in color, dependent on condition. The yellow bar represents the odor presentation period. The area under the curve (AUC) was calculated over the $5 \mathrm{sec}$ following odor onset. Boxes represent $25 \%$ and $75 \%$ quartiles, squares indicate means, and horizontal lines indicate medians. Whiskers show minimum and maximum values. Pre-to post-training effects were tested using Wilcoxon signed rank tests $\left({ }^{*} p<0.05 ;{ }^{* *} p<0.01\right)$. For $a, b, c, e, g$, and $h, n=10$ flies; for $d$ and $f, n=9$ flies.

\section{Figure 5}

\section{A supervised classifier can distinguish a conditioned from unconditioned odor when $\mathrm{Y} 1-\mathrm{MBON}$ is taken} into account. Calcium signals from pseudo-populations of all five MBON types ( $\gamma 1-\gamma 5)$ were used to train a machine learning-based classifier to distinguish pairs of odorants. (a) The accuracy of a supervised classifier in distinguishing between CS+ and CS- odors before and after classical conditioning ('pretraining' and 'trained') or randomly assigned designations of odor $A$ and $B$ to be differentiated in control conditions ('odor only' control and 'shock only' control). (b) The accuracy of the same supervised classifier in distinguishing between CS+ and CS- odors after classical conditioning if all $y$-lobe MBONs 
are included, or if one MBON type is removed from the training and test data sets. Bars represent means \pm SEM, $n=1000$ repetitions of MBON pseudo-populations. Significant difference from level of chance $(50 \%)$ determined by one sample Wilcoxon signed rank test $\left({ }^{* \star} \mathrm{p}<0.0001\right)$.

\section{Supplementary Files}

This is a list of supplementary files associated with this preprint. Click to download.

- HancockSupplementaryMaterial.docx 